\title{
Informe editorial, números 7 y 8 (2020-2021)
}

\author{
Jaime Escribano Pizarro y Antonio Valera Lozano
}

\section{CAMBIOS ANTE UN NÚMERO ESPECIAL: NUESTRO HOMENAJE AL PROFE. JOAN NOGUERA TUR}

Como es habitual, no podemos comenzar de otro modo que mostrando nuestra gratitud a todos los que han confiado en TERRA para dar salida a sus investigaciones, trabajos y experiencias profesionales en el ámbito del desarrollo local. Gracias a ellos, y al trabajo de todos los miembros del Consejo Editorial y del Consejo Asesor, es posible contar con dos números más en nuestra trayectoria, concretamente el que cerraba el año 2020, el número siete, primera vez que se publican dos números por año (uno cada semestre), y el que abre el presente 2021, el número ocho.

En relación al Consejo Editorial, varias son las novedades acontecidas desde julio de 2020 hasta la actualidad. Por un lado, dos cambios seguidos en la secretaría de la revista. Hasta agosto de 2020 nuestra compañera la Dra. Carme Melo era la responsable de dicha área, pero el 01 de septiembre tuvo que renunciar a su labor en TERRA debido a la responsabilidad que supone la dirección del Centro Internacional de Gandía de la Universidad de Valencia. Su lugar lo ocupó entonces el Profe. Dr. José Javier Serrano, pero solo por cinco meses, dado que el 31 de enero del presente año asumió la codirección del Máster de Cooperación al Desarrollo, también de la Universidad de Valencia. Por tanto, desde el 01 de febrero es el Dr. Antonio Valera, miembro del IIDL, el responsable de la secretaría de TERRA.

El Consejo Editorial se ha visto igualmente modificado por diversas razones, de modo que han causado baja nuestros compañeros el Profe. Joaquín Martín, del Dpto. de Derecho Constitucional, Ciencia Política y de la Administración (Universidad de Valencia), y la Profa. Berezi Elorrieta, del Centro Universitario Internacional de Barcelona. En su lugar, se han incorporado a este consejo la Profa. Mercedes Botija Yagüe, del Dpto. de Trabajo Social y Servicios Sociales (Universidad de Valencia), y la Profa. Andrea de Toni, del Dipartimento di Architettura e Studi Urbani (DAStU) del Politecnico di Milano (Italia). De este modo, entre los cambios acometidos en la secretaría y el consejo, mantenemos la misma proporción de sexos que la iniciada en febrero de 2019, momento en que tomamos la dirección de la revista, y además con la incorporación de la Dra. de Toni, logramos una mayor internacionalización del Consejo Editorial, ítem clave para los procesos de indexación en sellos de prestigio, como por ejemplo el de la FECYT.

La mayor novedad que podemos apuntar desde julio de 2020, se refiere a la distinción que incluimos en el presente número, el octavo, a modo de sección Homenaje dirigida a nuestro compañero el Dr. Joan Noguera Tur, jubilado oficialmente el 01 de enero de 2020 por enfermedad permanente. Profesor Titular del Dpto. de Geografía de la Universidad de Valencia, miembro del Instituto Interuniversitario de Desarrollo Local de Valencia 
desde su formación en septiembre de 2005, y Director del mismo desde marzo de $2012^{1}$ hasta el pasado diciembre de 2018, fue, aparte del principal promotor de que el IIDL temga una revista científica con personalidad propia y prestigio reconocido, TERRA, compañero y amigo de quien redacta estas líneas. Recuerdo perfectamente su labor como profesor, en materias como Geografía Regional de Europa de la Licenciatura de Geografía, durante el curso 2000-2001, pero también como investigador, puesto que coincidí con él varios años en la misma unidad de investigación, UDERVAL, tras comenzar mi Tesis Doctoral en 2004. Siempre estaba dispuesto a ayudar a cualquiera que lo necesitase, como fue mi caso en más de una ocasión con un consejo o una propuesta de trabajo, por ejemplo, la de participar como docente junto a él en una de mis primeras experiencias como tal ("Máster Visión Territorial y Sostenible del Desarrollo, Métodos e Instrumentos para la Acción Institucional", en el curso 2014-2015), o como (co)autor en la obra que en 2016 editó sobre desarrollo local dirigida, particularmente, al público latinoamericano y español: "La visión territorial y sostenible del desarrollo local. Una perspectiva multidisciplinar".

Figura 1. Call for papers para el número de TERRA en homenaje al Profe. Joan Noguera

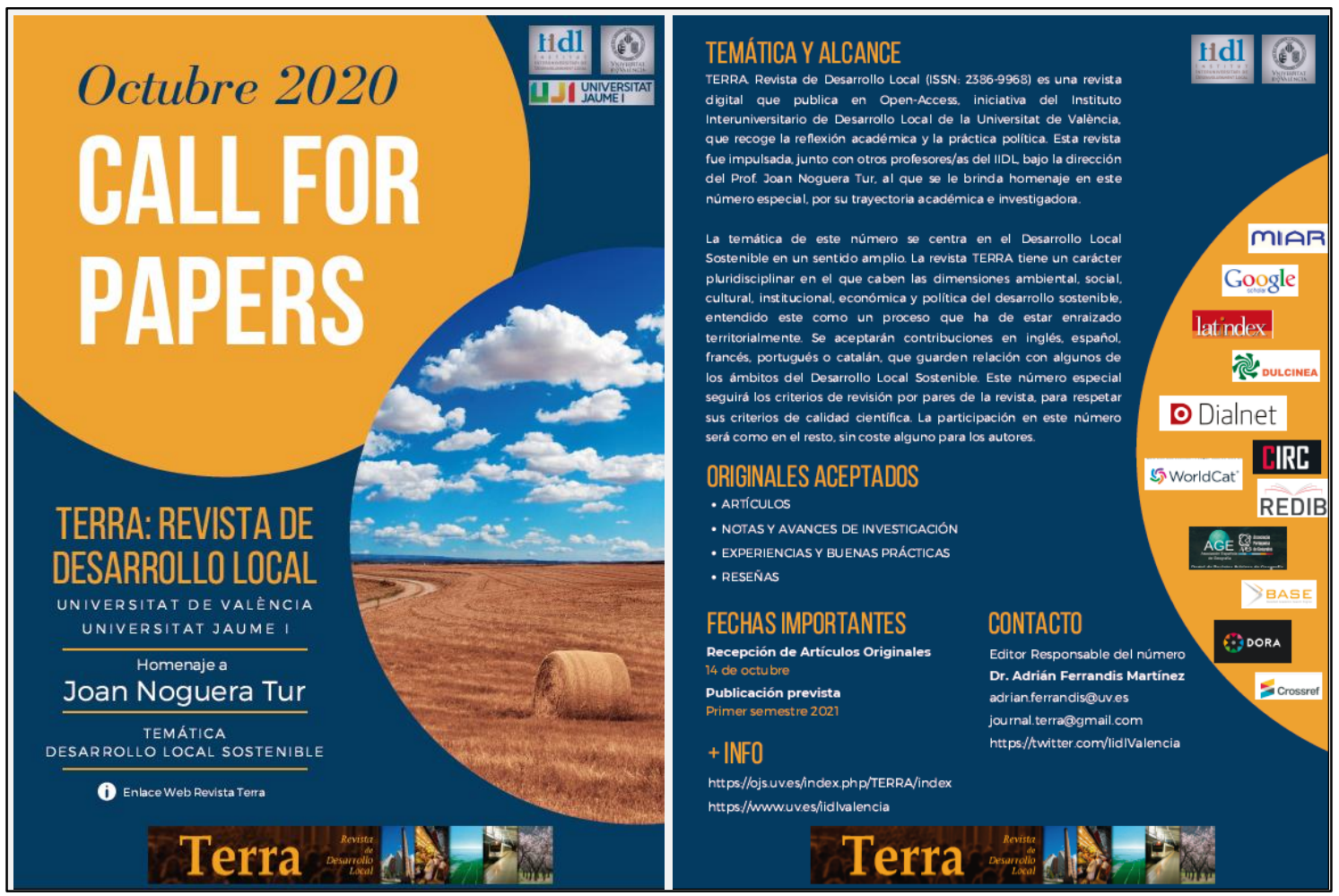

Fuente: Adrián Ferrandis y LOCSUS.

Desde el IIDL, pero en particular desde el grupo de investigación que en él formó Joan, LOCSUS, y concretamente por parte de su primer discípulo, el Profe. Dr. Adrián Ferrandis, este número alberga un humilde homenaje a su labor como investigador, pero también a su valor como persona (Figura 1). De ahí que, junto a aportaciones realizadas con independencia a este reconocimiento, contemos con numerosas contribuciones de compañeros y amigos, docentes e investigadores de la Universidad de Valencia, pero

\footnotetext{
${ }^{1}$ https://www.uv.es/uvweb/universidad/es/lista-noticias/profesor-joan-noguera-nuevo-director-delinstituto-interuniversitario-desarrollo-local-1285846070123/Noticia.html?id=1285851433805
} 
también de otras universidades españoles o extranjeras, con los que la carrera de Joan se entrecruzó en más de una ocasión. Y con quienes compartimos una misma sensación: no solo la repentina perdida de un profesional ejemplar, sino la injusta ausencia de un gran amigo, un hombre bueno, una mejor persona.

En total, de los 40 textos que conforman la presente publicación, la mayor cantidad de contribuciones aparecida hasta el momento en un único número de TERRA, 25 se corresponden con la aportaciones homenaje al Profe. Joan Noguera, concretamente 16 Artículos, tres Notas de Investigación, dos Buenas Prácticas, y cuatro Reseñas (Tabla 1). Para diferenciar todas ellas de las participaciones recibidas de forma "natural" por parte de la revista, desde el Consejo Editorial, junto con el Profe. Adrián Ferrandis, editor invitado en este número, se decidió que las destinadas al Profe. Joan Noguera contasen con una carátula distintiva conformada por una fotografía de Joan, y las palabras "Homenaje a Joan Noguera Tur" (Figura 2). Mientras, el resto mantendrían el formato habitual empleado en TERRA desde el número 5 (2019).

Figura 2. Ejemplo de carátula distintiva para las aportaciones en homenaje al Profe. Joan Noguera

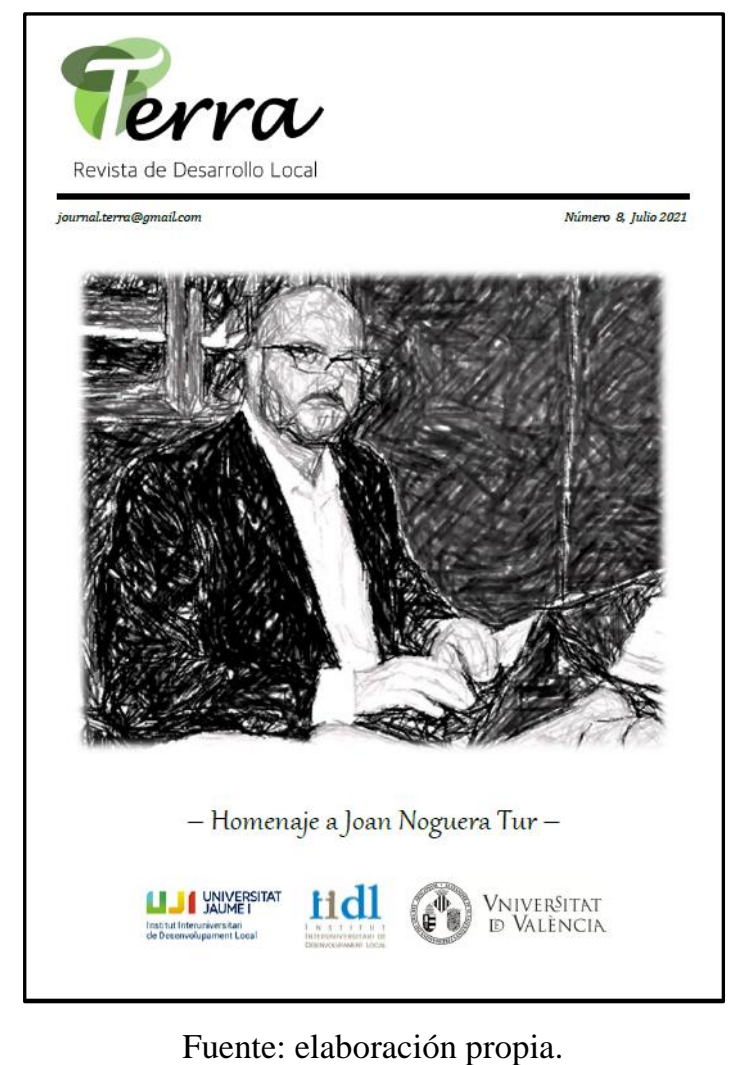

Las fotografías empleadas en las carátulas de apertura que diferencian las contribuciones dirigidas en homenaje a Joan, han sido aportadas por múltiples compañeros de trabajo, tanto presentes como pasados, con quienes Joan compartió proyectos de investigación, reuniones de trabajo, salidas de campo, o simplemente momentos distendidos de ocio en sus comisiones por España, Europa y Latinoamérica. Pero igualmente importante ha sido el aporte realizado por familiares y amigos, ajenos al mundo académico-universitario, pero que han querido compartir con todos nosotros sus recuerdos más preciados junto a Joan. A todos y todas, gracias. 
Tabla 1. Relación de contribuciones del número 8 en homenaje a Joan Noguera Tur

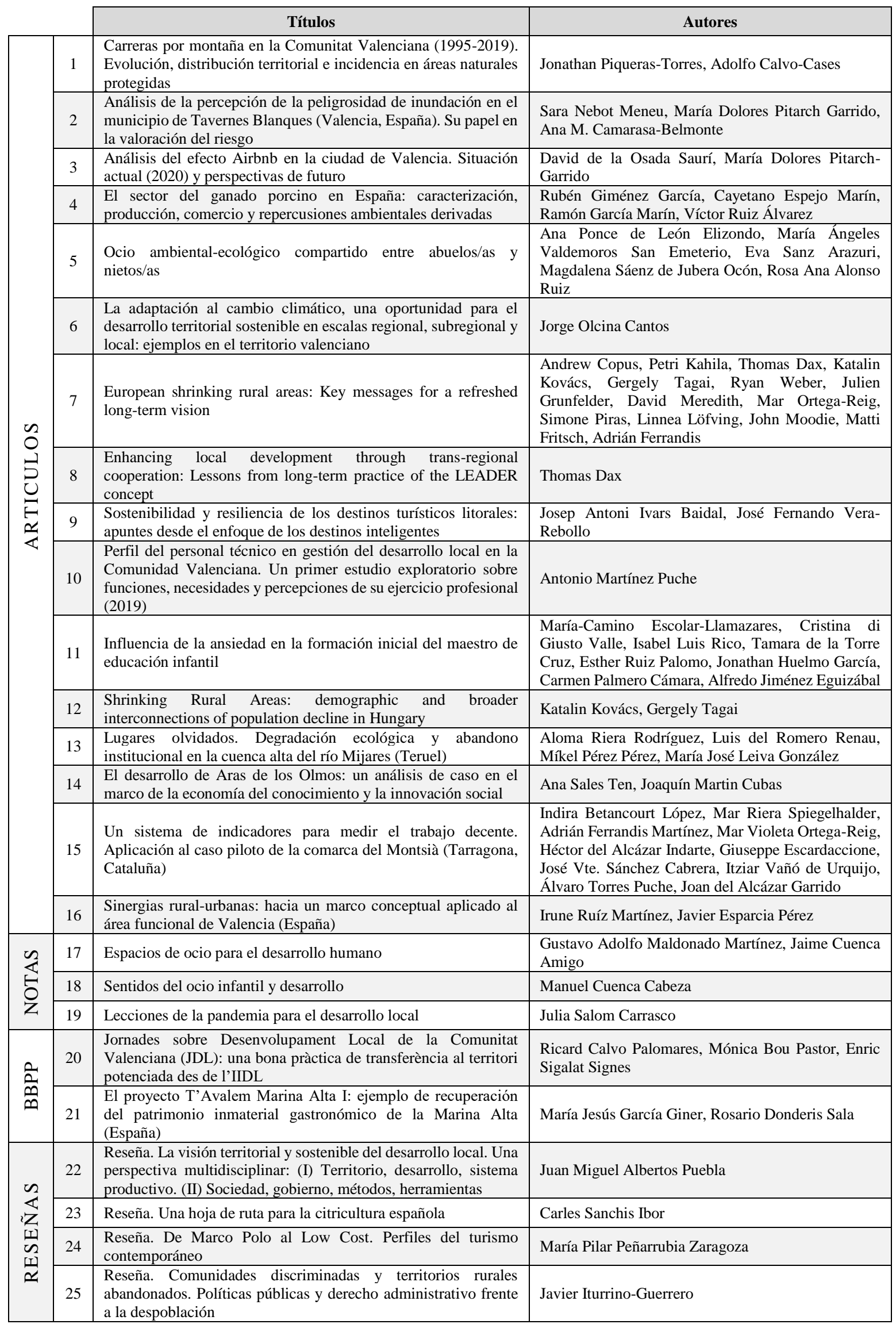

Fuente: elaboración propia. 


\section{REALIZACIONES Y TAREAS PENDIENTES}

Desde el último Informe editorial, publicado en verano de 2020 (número 6), hasta la actualidad, la regularidad en la recepción de originales se ha consolidado. Algunos, como artículos o notas de investigación, y otros directamente como reseñas o experiencias y buenas prácticas. Esta consolidación no solo se registra en cantidad, sino también en la calidad de los textos recibidos. En consecuencia, no podemos más que seguir aplicándolas como hasta ahora: la difusión de TERRA en el ámbito académico e investigador latinoamericano y español, a través de la participación en reuniones y/o jornadas científicas relacionadas con el Desarrollo Local; la aceptación de originales en distintos idiomas (español, catalán, portugués, inglés y francés); la actualización constante de la página web de la revista, en particular a través de la sección de textos pre-publicados; la invitación a proponer y participar en monográficos derivados de proyectos de investigación; la conexión con juniors investigadores interesados en conseguir dar salida a sus trabajos; la divulgación de los contenidos entre colectivos interesados en el ámbito del Desarrollo Local desde el mundo académico y/o profesional; etc.

Entre las realizaciones efectuadas en este último año, tenemos, primero, la actualización de los logos de TERRA, por parte del Profe. José Javier Serrano, durante el tiempo que estuvo al frente de la secretaría de la revista (Figura 3). La propuesta realizada, aparte del cambio cromático y de tipo de letra, se vincula a la unión de cada una de las cuatro áreas temáticas que organizan el IIDL en la Universidad de Valencia (Gobernanza Territorial, Ordenación del Territorio y Medio Ambiente; Desarrollo Local, Turismo y Economía de la Cultura; Cohesión Social), en los óvalos o elipses que enmarcan la inicial de la revista.

Figura 3. Evolución de los logos de TERRA. Antiguos y actuales

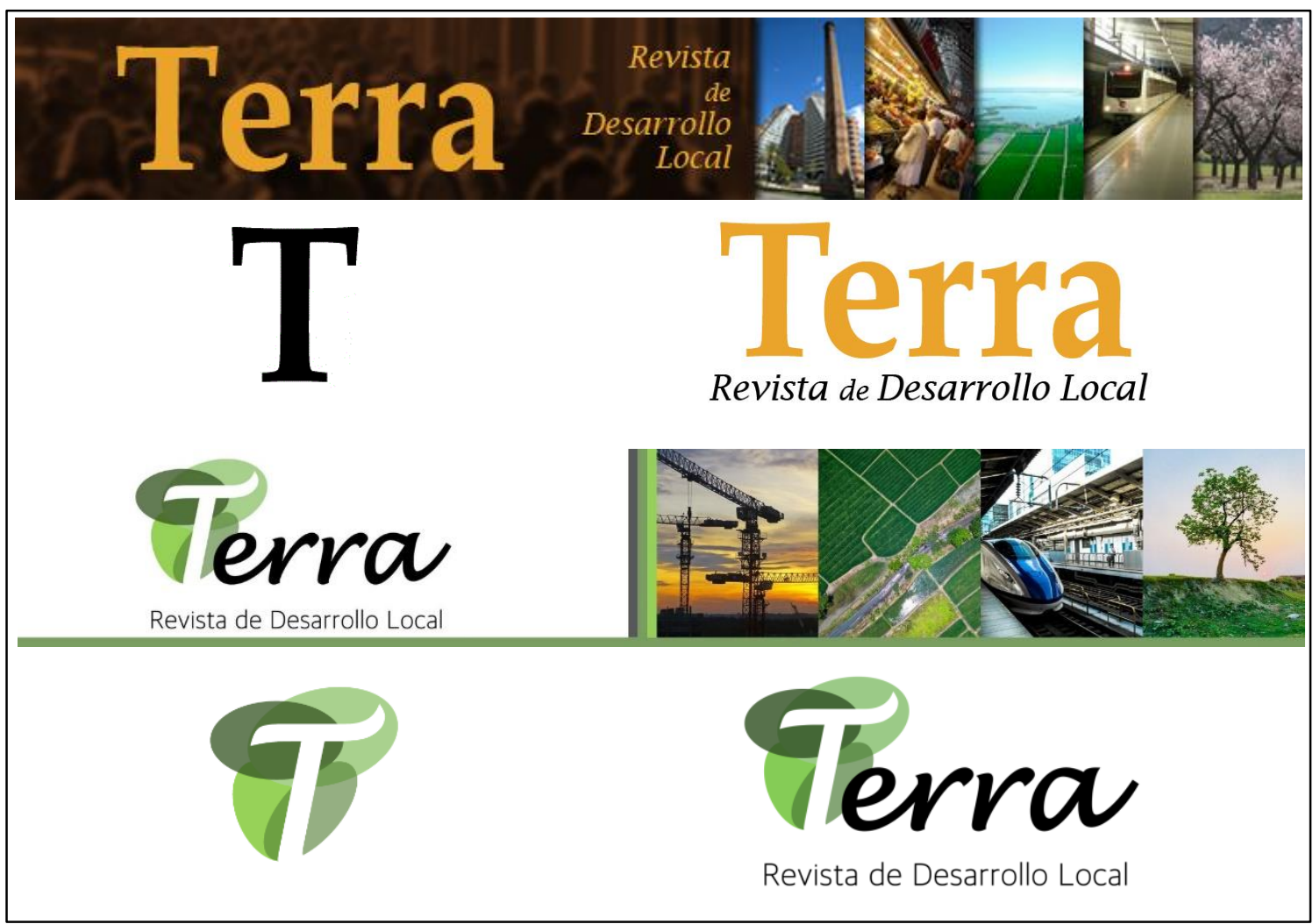

Fuente: elaboración propia. 
Durante este año también se ha abordado la actualización de las Normas de Publicación. Por un lado, en línea con las novedades de carácter voluntario incorporadas en junio de 2020 (ideas clave, resumen extendido en inglés y contribución según autores), y presentadas ya para el segundo número del año pasado (el séptimo) como obligatorias para los textos aceptados, exclusivamente, como artículos. Y, por otro lado, con otras incorporaciones más recientes (enero de 2021), como: i) la de atender al lenguaje inclusivo; ii) la posibilidad de ofrecer hasta cinco posibles evaluadores potenciales; y iii) la posibilidad de incorporar una "imagen de portada" que pudiera servir como portada del número en el que se publicase el texto en cuestión (solo en el caso de los textos aceptados como artículos), así como para la promoción de la revista en RRSS u otras vías (Tabla 2).

Tabla 2. Resumen de las Normas para Autores de TERRA

\begin{tabular}{|c|c|c|c|c|c|}
\hline & Extensión & Evaluación & Estructura & Contenidos & Formato \\
\hline$\frac{\mathscr{0}}{3}$ & $\begin{array}{c}8.000- \\
10.000 \\
\text { palabras }\end{array}$ & $\begin{array}{c}\text { Externa } \\
\text { por pares } \\
\text { ciegos }\end{array}$ & $\begin{array}{l}\text { 1. Título } \\
\text { 2. Resumen breve } \\
\text { (200 palabras) } \\
\text { 3. 3-5 palabras clave } \\
\text { 4. 3-5 ideas clave } \\
\text { 5. Resumen } \\
\text { extendido en } \\
\text { inglés } \\
\text { 6. Contribución de } \\
\text { autores } \\
\text { 7. Imagen de portada }\end{array}$ & $\begin{array}{l}\text { 1. Introducción y } \\
\text { justificación } \\
\text { 2. Bases teórico- } \\
\text { conceptuales } \\
\text { 3. Objetivos, } \\
\text { metodología y } \\
\text { fuentes } \\
\text { 4. Resultados } \\
\text { 5. Discusión } \\
\text { 6. Conclusiones } \\
\text { 7. Orientaciones futuras } \\
\text { 8. Referencias }\end{array}$ & \multirow{4}{*}{$\begin{array}{l}\text { 1. Página A4 (3 cm } \\
\text { en todos los } \\
\text { márgenes) } \\
\text { 2. Interlineado } \\
\text { sencillo } \\
\text { 3. Justificación } \\
\text { completa } \\
\text { 4. Times New } \\
\text { Roman tamaño } \\
\text { 12 puntos } \\
\text { 5. Los párrafos } \\
\text { irán sin sangrar } \\
\text { y la separación } \\
\text { entre los mismos } \\
\text { será de un } \\
\text { espacio } \\
\text { 6. Los } \\
\text { encabezamientos } \\
\text { han de ser } \\
\text { numéricos (ej.: } \\
\text { 2, 2.1., 2.1.1.) } \\
\text { 7. Los editores se } \\
\text { encargarán de la } \\
\text { numeración de } \\
\text { páginas } \\
\text { Más detalles: } \\
\text { ver desde el } \\
\text { apartado } 2 \text { de las } \\
\text { Normas para } \\
\text { Autores }\end{array}$} \\
\hline 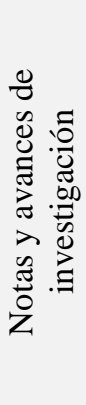 & $\begin{array}{c}6.000- \\
8.000 \\
\text { palabras }\end{array}$ & $\begin{array}{c}\text { Externa } \\
\text { por pares } \\
\text { ciegos }\end{array}$ & $\begin{array}{l}\text { 1. Título } \\
\text { 2. Resumen breve } \\
\text { ( } 200 \text { palabras) } \\
\text { 3. 3-5 palabras } \\
\text { clave } \\
\text { 4. Contribución de } \\
\text { autores }\end{array}$ & $\begin{array}{l}\text { 1. Introducción y } \\
\text { justificación } \\
\text { 2. Bases teórico- } \\
\text { conceptuales } \\
\text { 3. Objetivos, } \\
\text { metodología y } \\
\text { fuentes } \\
\text { 4. Resultados } \\
\text { 5. Conclusiones } \\
\text { 6. Orientaciones futuras } \\
\text { 7. Referencias }\end{array}$ & \\
\hline 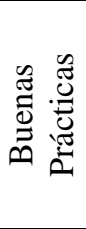 & $\begin{array}{c}2.000- \\
6.000 \\
\text { palabras }\end{array}$ & $\begin{array}{l}\text { Comité } \\
\text { Editorial }\end{array}$ & $\begin{array}{l}\text { 1. Título } \\
\text { 2. Resumen breve } \\
\text { (200 palabras) } \\
\text { 3. 3-5 palabras clave }\end{array}$ & $\begin{array}{l}\text { 1. Introducción } \\
\text { 2. Desarrollo } \\
\text { 3. Resultados } \\
\text { 4. Conclusiones } \\
\text { 5. Referencias (si fueran } \\
\text { necesarias) }\end{array}$ & \\
\hline 胥 & $\begin{array}{c}2.000- \\
4.000 \\
\text { palabras }\end{array}$ & $\begin{array}{l}\text { Comité } \\
\text { Editorial }\end{array}$ & $\begin{array}{l}\text { 1. Título } \\
\text { 2. Resumen breve } \\
\text { (200 palabras) } \\
\text { 3. 3-5 palabras clave }\end{array}$ & $\begin{array}{l}\text { Libre / A establecer } \\
\text { por el/la autor/a }\end{array}$ & \\
\hline
\end{tabular}

Fuente: elaboración propia.

También, durante el último trimestre de 2020, se llevó a cabo la traducción al inglés y al catalán/valenciano, de todas y cada una de las secciones y documentos on-line disponibles para uso y/o consulta existentes en la web de la revista (como el caso de las Normas para Autores, el Formulario para la Evaluación Externa, etc.) (Figura 4). Para facilitar la identificación de cada idioma, dadas las características técnicas del portal en el que se aloja TERRA, se optó por emplear una escala cromática diferenciada: en color negro, español, en color azul, inglés, y en color marrón, catalán/valenciano. 
Figura 4. Detalle de la página inicial de TERRA en español, inglés y catalán/valenciano

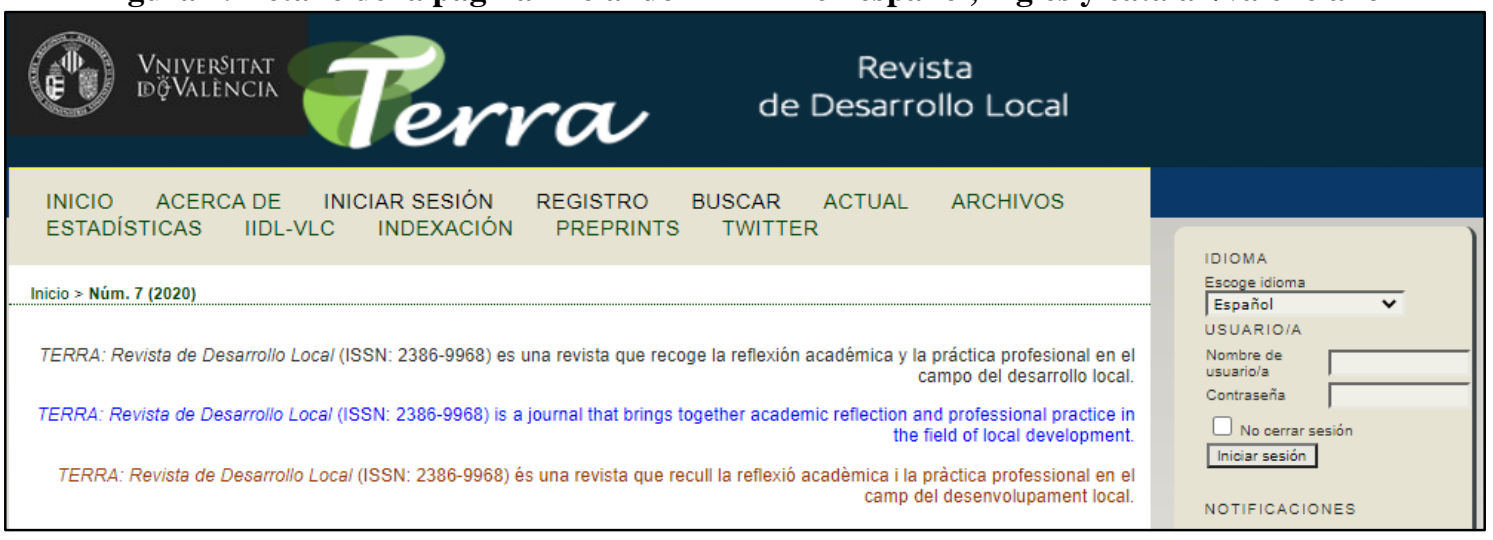

Fuente: https://ojs.uv.es/index.php/TERRA

Otro conjunto de realizaciones efectuadas es la tramitación de solicitudes para la incorporación de TERRA en nuevas bases bibliográficas y la adhesión a sellos de reconocimiento por una investigación de calidad (Tabla 3).

Tabla 3. Presencia de TERRA en bases bibliográficas, plataformas de evaluación y directorios (2015-2021)

\begin{tabular}{|c|c|c|c|c|}
\hline & $\begin{array}{c}\text { Antes de } \\
\text { febrero de 2019 }\end{array}$ & $\begin{array}{c}\text { De febrero a } \\
\text { julio de 2019 }\end{array}$ & $\begin{array}{c}\text { De julio a } \\
\text { julio de 2020 }\end{array}$ & De julio a julio de 2021 \\
\hline Dialnet Catálogo & $\mathrm{X}$ & & & \\
\hline MIAR & & $\mathrm{X}$ & & \\
\hline Google Scholar & & $\mathrm{X}$ & & \\
\hline Latindex Directorio & & $\mathrm{X}$ & & \\
\hline Dulcinea & & & Categoría D & \\
\hline CIRC & & & $\mathrm{X}$ & \\
\hline Redib & & & $\mathrm{X}$ & \\
\hline $\begin{array}{c}\text { Portal de Revistas } \\
\text { Ibéricas de Geografía }\end{array}$ & & & $\mathrm{X}$ & \\
\hline $\begin{array}{c}\text { DORA (Declaración } \\
\text { de San Francisco) }\end{array}$ & & & $\mathrm{X}$ & \\
\hline BASE & & & $\mathrm{X}$ & \\
\hline Crossref & & & $\mathrm{X}$ & \\
\hline WorldCat & & & & Q2 en Economía (2019) y \\
\hline Dialnet Métricas & & & & Cumple 34 de 38 criterios \\
\hline Latindex Catálogo 2.0 & & & & $\mathrm{X}$ \\
\hline ERIH PLUS & & & & $\mathrm{X}$ \\
\hline EcoLint & & & & $\mathrm{X}$ \\
\hline DOAJ & & & & \\
\hline Dimensions & & & & \\
\hline
\end{tabular}

Fuente: https://ojs.uv.es/index.php/TERRA/about/editorialPolicies\#custom-2

La mayor parte de estas realizaciones han sido posible gracias a los 500,00 euros de apoyo económico otorgados en 2020, y a los 800,00 euros destinados el presente año por parte de la dirección de la sede de la Universitat de València del IIDL, que evidencia así su 
decidida apuesta por la consolidación de TERRA como una revista científica de calidad contrastada. En concreto, los 500,00 euros concedidos durante el año 2020 se emplearon en establecer con ADEIT unas Prácticas Externas Extracurriculares por un total de 150 horas, finalmente desempeñadas por Dña. Águeda Segarra Áles durante todo el mes de septiembre. Por su parte, del presupuesto de 2021 se han empleado ya 400,00 en una estrategia similar, ofertar unas Prácticas Externas Extracurriculares vía ADEIT, por un total de 120 horas entre el 01 de julio y el 30 de septiembre, ofrecidas a D. Francisco Cuello Llobell.

En cuanto a las tareas pendientes y previstas realizar de aquí a julio de 2022, mantenemos algunas heredadas del año anterior (con ciertos matices), e incorporamos otras nuevas:

a) Conseguir formar parte de las Bases de Datos y Directorios de Publicación Periódicas EBSCO, ProQuest, ELSERVIER, InDICEs del CSIC, Agenzia Nazionale di Valutazione del Sistema Universitario e della Ricerca, RESH (Revistas Españolas de Ciencias Sociales y Humanidades), Journal Scholar Metrics, Carhus Plus +, Dimensions, y valorar la posibilidad de participar en los procesos de evaluación relativos al sello de la FECYT y a la plataforma ESCI (Emerging Sources Citation Index, de Web Of Science), con tal de conocer con exactitud los ítems a cumplir y sobre los que trabajar en los próximos años.

b) Cambiar la estrategia de abrir un perfil propio en redes sociales como, por ejemplo, Twitter o Facebook, por rentabilizar más y mejor el hecho de estar integrados ya en las cuentas del IIDL de las sedes de la Universitat de València y de la Universitat Jaume I de Castelló. Esta decisión supone postergar para medio o largo plazo disponer de un perfil particular con el que optar a otros indicadores de citación, como es el caso de ALTMETRICS ${ }^{2}$, cada vez más empleado en el ámbito de las revistas científicas. Sin embargo, el coste de mantener activo un perfil de este tipo, y sobre todo, hacerlo de forma adecuada (tras las consultas realizadas, con tres o más tweets originales por día, no retweets o acciones similares), sobrepasa ampliamente las posibilidades de gestión real de los miembros de la dirección y secretaría de la revista.

c) Consolidar la participación de toda la oferta formativa del IIDL en la dinámica de TERRA, en particular, como fuente de potenciales autores y/o revisores, completando así la colaboración actualmente existente en este sentido, procedente de dicha institución u otras similares.

d) Continuar con la búsqueda de cooperación con cátedras institucionales-universitarias con evidente impacto en el estudio del Desarrollo Local, existentes en cualquier centro universitario español y/o latinoamericano.

e) Establecer vías de colaboración con asociaciones profesionales en el ámbito del Desarrollo Local radicadas en la Comunidad Valenciana o fuera de ella, por ejemplo, FEPRODEL (Federación de Profesionales del Desarrollo Local), ADLYPSE (Federación de Personal Técnico en Gestión del Desarrollo Local), REDEL (Red de Entidades para el Desarrollo Local), RDR/DLS (Red de Redes de Desarrollo Local Sostenible), etc. Y, también, con colectivos con idénticos intereses procedentes del ámbito académico o entidades colegiadas, como es el Grupo de Trabajo de Desarrollo Local de la Asociación Española de Geografía (AGE), el Colegio Oficial de Politólogos y Sociólogos, el Colegio Oficial de Geógrafos, etc.

\footnotetext{
${ }^{2}$ El comentario o discusión de un artículo puede ser visto como una métrica que muestra el impacto potencial del texto en cuestión. Las fuentes típicas de los datos para el cálculo de este indicador incluyen Facebook, Google+, Twitter, blogs científicos y páginas de Wikipedia.
} 


\section{RESULTADOS}

Los resultados obtenidos de todas las acciones descritas en el apartado anterior sobre realizaciones, se sintetizan bien con los datos relativos al número de textos publicados en el número actual, el octavo, sin distinguir si se trata de originales que terminan como artículos, notas, buenas prácticas o reseñas: 40 en total. Lógicamente, de este conjunto interesa conocer los que terminan como artículos y/o notas de investigación, puesto que son las contribuciones que realmente se tienen en cuenta para el cálculo de indicadores de calidad, como por ejemplo los relativos al sello de la FECYT. Si atendemos a estos dos tipos de aportaciones, obtenemos unos resultados que satisfactorios para una publicación todavía por indexar en las principales plataformas de referencia como la mencionada FECYT, ESCI o SCOPUS, entre otras (Tabla 4).

Tabla 4. Datos relativos a originales recibidos como artículos/notas para los números 7 y

\begin{tabular}{|c|c|c|c|c|}
\hline & \multicolumn{2}{|c|}{ Núm. 7 } & \multicolumn{2}{c|}{ Núm. 8 } \\
& Del 01 de julio al 31 de diciembre de 2020 & Del 01 de enero al 30 de junio de 2021 \\
\hline Recibidos & 19 & $100 \%$ & 19 & $100 \%$ \\
\hline Aceptados & 14 & $74 \%$ & 19 & $100 \%$ \\
\hline Rechazados & 5 & $26 \%$ & 0 & $0 \%$ \\
\hline
\end{tabular}

Fuente: elaboración propia a partir de los datos de gestión de TERRA.

El número 7 (relativo a la segunda mitad de 2020) obtuvo también unos buenos resultados, en particular, en el contexto de pandemia COVID19, fenómenos que afecto notablemente a las capacidades de muchos investigadores e investigadoras para llevar a cabo sus trabajos y estudios, en un entorno de confinamiento y con adversas situaciones de conciliación laboral-familiar ${ }^{3}$ (Tabla 5).

Tabla 5. Publicaciones en TERRA por sección y año de publicación

\begin{tabular}{|c|c|c|c|c|c|c|c|c|c|}
\hline & \multicolumn{4}{|c|}{ Datos absolutos } & \multicolumn{4}{c|}{ En porcentaje } \\
\hline & Artículos & Notas & BBPP & Reseñas & Total & Artículos & Notas & BBPP & Reseñas \\
\hline Número 1 (2015) & 4 & 0 & 1 & 0 & $\mathbf{5}$ & 80 & 0 & 20 & 0 \\
\hline Número 2 (2016) & 5 & 0 & 1 & 0 & $\mathbf{6}$ & 83 & 0 & 17 & 0 \\
\hline Número 3 (2017) & 7 & 0 & 0 & 0 & $\mathbf{7}$ & 100 & 0 & 0 & 0 \\
\hline Número 4 (2018) & 4 & 0 & 1 & 0 & $\mathbf{5}$ & 80 & 0 & 20 & 0 \\
\hline Número 5 (2019) & 6 & 1 & 2 & 0 & $\mathbf{9}$ & 67 & 11 & 22 & 0 \\
\hline Número 6 (2020) & 9 & 2 & 4 & 10 & $\mathbf{2 5}$ & 36 & 8 & 16 & 40 \\
\hline Número 7 (2020) & 10 & 1 & 3 & 7 & $\mathbf{2 1}$ & 48 & 5 & 14 & 33 \\
\hline Número 8 (2021) & 21 & 4 & 2 & 13 & $\mathbf{4 0}$ & 53 & 10 & 5 & 32 \\
\hline Totales & $\mathbf{6 6}$ & $\mathbf{8}$ & $\mathbf{1 4}$ & $\mathbf{3 0}$ & $\mathbf{1 1 8}$ & $\mathbf{5 6}$ & $\mathbf{7}$ & $\mathbf{1 2}$ & $\mathbf{2 5}$ \\
\hline
\end{tabular}

Fuente: elaboración propia a partir de: https://ojs.uv.es/index.php/TERRA/issue/archive. Se excluyen los "Informes editoriales", publicados habitualmente en el número de julio desde 2019.

\footnotetext{
${ }^{3}$ El COVID-19 limita más la investigación académica de las mujeres que la de los hombres, de Carlota García Sánchez (11 de junio de 2021). Disponible en: https://amecopress.net/El-COVID-19-limita-mas-lainvestigacion-academica-de-las-mujeres-que-la-de-los-hombres-23502. Recuperado el 23 de julio de 2021.
} 
Aparte de lograr el cierre del número actual con la cantidad de textos publicada más elevada y diversificada hasta la fecha (mantenemos la presencia de originales en todas las secciones de la revista), otro de los resultados que evidencian un impacto relativamente positivo de las acciones desarrolladas este último año, se relaciona con el número de visitas a la web de la revista registradas desde el 01 de julio de 2020 al 30 de junio de 2021, y con la cantidad de textos descargados en idéntico período (Tabla 6).

Este incremento tanto en el número de originales recibidos y publicados, en todas las secciones, como de visitas y de descargas registradas, concuerda igualmente con el aumento experimentado de las citas que han recibido los textos publicados en los números anteriores de la revista (Tabla 7).

Tabla 6. Consultas a la web de TERRA (visitas y descargas) de 2018 a 2021

\begin{tabular}{|l|c|c|c|c|c|}
\hline & $\begin{array}{c}\text { Visitas } \\
\text { (absolutas) }\end{array}$ & $\begin{array}{c}\text { Descargas } \\
\text { (absolutas) }\end{array}$ & $\begin{array}{c}\text { Visitas } \\
\text { al mes }\end{array}$ & $\begin{array}{c}\text { Descargas } \\
\text { al mes }\end{array}$ & $\begin{array}{c}\text { Una descarga cada } \\
\text { “...” visitas }\end{array}$ \\
\hline $01 / 01 / 2018-31 / 01 / 2019(13$ meses) & 3.967 & 2.487 & 305 & 191 & 1,6 \\
\hline $01 / 02 / 2019-30 / 06 / 2019(5$ meses $)$ & 2.349 & 1.986 & 470 & 397 & 1,2 \\
\hline $01 / 07 / 2019-30 / 06 / 2020(12$ meses $)$ & 7.629 & 6.328 & 636 & 527 & 1,2 \\
\hline $01 / 07 / 2020-30 / 06 / 2021(12$ meses) & 14.001 & 13.469 & 1.167 & 1.122 & 1,0 \\
\hline
\end{tabular}

Fuente: elaboración propia a partir de: https://ojs.uv.es/index.php/TERRA/statistics.

Tabla 7. Métricas de TERRA en Google Académico

\begin{tabular}{|l|c|c|c|}
\hline & A 23 de julio de 2019 & A 23 de julio de 2020 & A 23 de julio de 2021 \\
\hline Citas recibidas & 26 & 59 & 99 \\
\hline Índice H & 3 & 4 & 6 \\
\hline Índice i10 & 1 & 2 & 2 \\
\hline $\begin{array}{l}\text { Número máximo de citas } \\
\text { recibidas por un texto (*) }\end{array}$ & 13 & 18 & 25 \\
\hline Número de textos citados & 4 & 12 & 22 \\
\hline
\end{tabular}

Fuente: elaboración propia a partir de: https://scholar.google.es/citations?user=T3zUOrIAAAAJ\&hl=es. Nota (*): se trata del artículo titulado Bioregionalismo: una ventana hacia el desarrollo territorial endógeno y sustentable (Boisier, 2015).

Por finalizar, el último ejemplo que evidencia la adecuada estrategia adoptada por TERRA en su crecimiento, proviene de la internacionalización de la revista, entendida esta como el número de contribuciones publicadas en idioma distinto de español. En este sentido, consideramos tanto los textos presentados en inglés, francés o portugués, como los redactados en catalán/valenciano, idioma cooficial de la Universitat de València y de la Universitat Jaume I de Castelló y, por extensión, del IIDL, responsable último de la edición de TERRA (Tabla 8). Tanto el número 7 como el número 8 han recogido varias participaciones en otros idiomas, lo que ha permitido doblar con holgura el porcentaje de textos logrado ya en el primer número del año pasado, el sexto. En el caso del número actual, el octavo, en parte este resultado encuentra explicación en la participación de académicos de fuera de España en el homenaje realizado a Joan Noguera. Sin duda, esto supone un reto para los próximos números, en tanto en cuanto desde la dirección de la revista quisiéramos mantener, como mínimo, la media alcanzada en estos ocho números (es decir, una contribución en torno al 7\% de textos en idioma distinto del español). 
Tabla 8. Internacionalización de la revista a partir de los originales publicados

\begin{tabular}{|c|c|c|c|c|c|c|c|}
\hline \multirow{2}{*}{ Números } & \multirow{2}{*}{$\begin{array}{c}\text { Total textos } \\
\text { publicados }\end{array}$} & \multicolumn{5}{|c|}{ Originales distintos al español por sección } \\
\cline { 3 - 8 } & Artículos & Notas & BBPP & Reseñas & Totales & \% \\
\hline Número 1 (2015) & 5 & $1(\mathrm{i})$ & 0 & 0 & 0 & $\mathbf{1}$ & $\mathbf{2 0}$ \\
\hline Número 2 (2016) & 6 & 0 & 0 & 0 & 0 & $\mathbf{0}$ & $\mathbf{0}$ \\
\hline Número 3 (2017) & 7 & 0 & 0 & 0 & 0 & $\mathbf{0}$ & $\mathbf{0}$ \\
\hline Número 4 (2018) & 5 & 0 & 0 & 0 & 0 & $\mathbf{0}$ & $\mathbf{0}$ \\
\hline Número 5 (2019) & 9 & 0 & 0 & 0 & 0 & $\mathbf{0}$ & $\mathbf{0}$ \\
\hline Número 6 (2020) & 25 & $1(\mathrm{v})$ & 0 & 0 & 0 & $\mathbf{1}$ & $\mathbf{4}$ \\
\hline Número 7 (2020) & 21 & $1(\mathrm{i})$ & 0 & $1(\mathrm{v})$ & 0 & $\mathbf{2}$ & $\mathbf{9}$ \\
\hline Número 8 (2021) & 40 & $3(\mathrm{i})$ & 0 & $1(\mathrm{v})$ & 0 & $\mathbf{4}$ & $\mathbf{1 0}$ \\
\hline Totales & $\mathbf{1 1 8}$ & $\mathbf{6}$ & $\mathbf{0}$ & $\mathbf{2}$ & $\mathbf{0}$ & $\mathbf{8}$ & $\mathbf{7}$ \\
\hline
\end{tabular}

Fuente: elaboración propia a partir de: https://ojs.uv.es/index.php/TERRA/issue/archive. Notas: (i) inglés;

(v) valenciano/catalán.

Por último, recogemos a continuación el listado de evaluadores externos que han actuado desde el 01 de julio de 2020 hasta el 30 de junio de 2021, y que han contribuido a que TERRA se presente como una revista que cumple con todos los niveles de exigencia propios de las publicaciones basadas en la selección de originales para su publicación, a partir de un proceso de evaluación por pares ciegos. Desde la dirección de la revista les agradecemos su inestimable colaboración a nuestro crecimiento, realizando una labor muchas veces desapercibida y, aún, poco reconocida y valorada curricularmente.

1. Agustín Miquel Bartual. Scuola Sant'Anna di Pisa (Italia)

2. Albert Mora Castro. Universidad de Valencia

3. Alejandra Toscana. Universidad Nacional Autónoma de México (México)

4. Alejandro Pérez Cueva. Universidad de Valencia

5. Alicia Susana Ezeta Genis. Universidad Nacional Autónoma de México (México)

6. Ana Espinosa Seguí. Universidad de Alicante

7. Anna Fernández Poncela. Universidad Autónoma Metropolitana (México)

8. Antonini de Jiménez. Universidad Católica de Pereira (Colombia)

9. Antonio Prieto Cerdán. Universidad de Alicante

10. Arsenio Villar Lama. Universidad de Sevilla

11. Beatriz Rosas Rodríguez. Universidad Autónoma de Queretaro (México)

12. Carles Donat Muñoz. Universitat Autònoma de Barcelona

13. Carlos Cortes Samper. Universidad de Alicante

14. Carlos Uriel del Carpio Penagos. Universidad de Ciencias y Artes de Chiapas (México)

15. Carme Melo Escrihuela. Universidad de Valencia

16. Catalina Mahé Duque. Universidad Nacional de Colombia (Colombia)

17. Cayetano Espejo Marín. Universidad de Murcia

18. Daniel Herrera. Universidad de Oviedo

19. David Giner Sánchez. Universidad de Alicante

20. Diego García Monteagudo. Universidad de Valencia

21. Elia Fernández Díaz. Universidad de Cantabria

22. Eliseo Martínez Muñoz. Universidad de Valencia

23. Emilio Iranzo García. Universidad de Valencia

24. Ester González Herreros. Universidad de Alicante

25. Fabio Schwab do Nascimento. Universidad de Córdoba

26. Francisco Antonio Navarro Valverde. Universidad de Granada

27. Francisco Cebrián Abellán. Universidad de Castilla-La Mancha

28. Francisco Guevara Hernández. Universidad Autónoma de Chiapas (México) 
29. Frank Babinger. Universidad Complutense de Madrid

30. Giancarlo Cotella. Politécnico di Torino (Italia)

31. Giuseppe Lo Brutto. Benemérita Universidad Autónoma de Puebla (México)

32. Hilda Guerrero-García-Rojas. Universidad Michoacana de San Nicolás de Hidalgo (México)

33. Isaac Enríquez Pérez. Universidad Nacional Autónoma de México (México)

34. Isabel Marín Sánchez. Universidad de Granada

35. Isabelle Hillenkamp. Centre d'Etudes en Sciences Sociales sur les Mondes Africains, Américains et Asiatiques (Francia)

36. Itxaso Ruiz. Basque Centre for Climate Change

37. Iván Portugués Mollá. Universidad de Valencia

38. Jason Begley. Coventry University (Reino Unido)

39. Jonatan Arias García. Universidad de Granada

40. Jorge Hervás Más. Universidad Politécnica de Valencia

41. Jorge Olcina. Universidad de Alicante

42. José Ángel Llorente Adán. Profesor-Tutor de la Universidad Nacional de Educación a Distancia (UNED)

43. José Antonio Segrelles Serrano. Universidad de Alicante

44. José Daniel Anido Rivas. Universidad de Los Andes (Venezuela)

45. José Javier Serrano Lara. Universidad de Valencia

46. José Navarro Pedreño. Universidad de Murcia

47. Josep Vicent Boira i Maiques. Universidad de Valencia

48. Josep Vicent Pérez Cosín. Universidad de Valencia

49. Juan Antonio Márquez Domínguez. Universidad de Huelva

50. Juan Bautista Ferreres Bonfill. Universitat Jaume I de Castelló

51. Juan Carlos Delgado Barrios. Universidad de Los Andes (Venezuela)

52. Juan Carlos García Palomares. Universidad de Valencia

53. Juan Carlos Maroto. Universidad de Granada

54. Juan Rivero Rodríguez. Universidad Autónoma de Madrid

55. Juana Hernández Chavarría. Facultad Latinoamericana de Ciencias Sociales (México)

56. Julio Pereiro. Universidad Nacional del Centro de la Provincia de Buenos Aires (Argentina)

57. Luis Salinas Arreortua, Universidad Nacional Autónoma de México (México)

58. Manuel Santana Turégano. Universidad de La Laguna

59. Mar Riera Spiegelhalder. Universidad Europea de Valencia

60. María Albert Rodrigo. Universidad de Valencia

61. María del Carmen Rodríguez Rodríguez. Universidad de A Coruña

62. María Esther Ayala Arcipreste. Universidad Autónoma de Campeche (México)

63. María Pilar Peñarrubia Zaragoza. Universidad de Valencia

64. Marta Climent. Universidad de Valencia

65. Moisés Simancas Cruz. Universidad de La Laguna

66. Néstor Vercher Savall. Universidad de Valencia

67. Oleg Lazovski Bakhirev. Universidad de Santiago de Compostela

68. Olmes García Bode. Universidad Las Tunas (Perú)

69. Pilar Almoguera Sallent. Universidad de Sevilla

70. Rubén Arnandis i Agramunt. Universidad de Valencia

71. Vicente Estruch Guitart. Universidad Politécnica de Valencia

72. Xavier Amat Montesinos. Universidad de Alicante

Valencia, 27 de julio de 2021

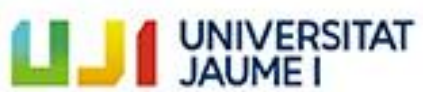

Institut Interuniversitari de Desenvolupament Local

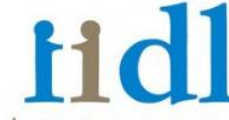

I N S T I T U T INTERUNIVERSITARI DE

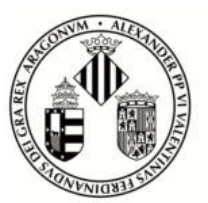

VNIVERSITAT (E) VAlÈnCIA 